\title{
Determination of Optimum Thermalization Distance Based on Trade-off Relationship between Resolution, Line Edge Roughness, and Sensitivity of Chemically Amplified Extreme Ultraviolet Resists
}

\author{
Takahiro Kozawa ${ }^{a, b^{*}}$ and Seiichi Tagawa $a^{a, b}$ \\ ${ }^{a}$ The Institute of Scientific and Industrial Research, Osaka University, \\ 8-1 Mihogaoka, Ibaraki, Osaka 567-0047, Japan \\ bapan Science and Technology Agency, CREST, \\ clo Osaka University, 8-1 Mihogaoka, Ibaraki, Osaka 567-0047, Japan \\ *E-mail address: kozawa@sanken.osaka-u.ac.jp
}

\begin{abstract}
In chemically amplified extreme ultraviolet (EUV) resists, the thermalization distance of secondary electrons is a major cause of the degradation of the original EUV aerial image and increases the line edge roughness (LER). However, the longer the thermalization distance is, the more acid generators are decomposed. As the acid concentration increases, the acid diffusion length required for the polarity change of resist polymers decreases. With the reduction in diffusion length, the resolution blur induced by acid diffusion is suppressed. In this study, we investigated the optimum thermalization distance for obtaining the best latent image quality. The optimum thermalization distance for $32 \mathrm{~nm}$ line-and-space patterns was $7 \mathrm{~nm}$ at $10 \mathrm{wt} \%$ acid generator (TPS-tf) concentration. The optimum thermalization distance decreased with the half-pitch of line-and-space patterns. For $11 \mathrm{~nm}$ half-pitch, the optimum thermalization distance was 3 $\mathrm{nm}$, which is approximately the same as that in the poly(4-hydroxystyrene) matrix.
\end{abstract}

Keywords: EUV lithography, chemically amplified resist, line edge roughness, thermalization distance, trade-off

\section{Introduction}

The concept of "chemical amplification" devised by Ito and Willson has been an indispensable technology in semiconductor manufacturing since the transition of exposure tools from the $\mathrm{i}$ line of a $\mathrm{Hg}$ lamp to a $\mathrm{KrF}$ excimer laser.[1] However, the performance of chemically amplified resists approaches its limit with the reduction in feature size. In particular, the trade-off relationship between resolution, line edge roughness (LER), and sensitivity (RLS trade-off relationship) is the most serious problem in the next-generation lithography.[2] In chemically amplified resists, the aerial image of incident photons is converted to an acid image. The quality of the acid image is an important issue from the viewpoint of the RLS trade-off relationship.[3]

After ArF immersion lithography with double patterning technology, extreme ultraviolet (EUV) lithography is expected to be applied to high-volume production.[4-6] The acid generation mechanisms of EUV resists have been investigated.[7] After EUV photons are absorbed by resist molecules, photoelectrons are emitted with an energy of $\sim 80 \mathrm{eV}$. The photoelectrons further induce the ionization and excitation of molecules. The 4.2 electrons are generated by a single EUV photon on average. The ejected electrons lose their kinetic energy upon their interaction with the surrounding molecules and are eventually decelerated down to thermal energy. Acid generator molecules are decomposed through their reaction with the thermalized electrons.[8] The typical concentration of an acid generator, for example, triphenylsulfonium triflate (TPS-tf), is 0.16 molecule $\mathrm{nm}^{-3}$ (10 $\mathrm{wt} \%$ relative to the resist polymer). Thus, the average distance between the nearest acid generators is $\sim 2 \mathrm{~nm}$. On the other hand,

Received March 30, 2011

Accepted May 11, 2011 
EUV photons are almost randomly absorbed by resist-constituent molecules. The inelastic mean free path of photoelectrons and secondary electrons is less than $1 \mathrm{~nm}$ in the energy range from the ionization potential of resist materials to the energy of EUV photons (92.5 eV).[9] The energy deposition of photoelectrons and secondary electrons is also a random process. Nevertheless, the quantum efficiency of acid generation through EUV sensitization exceeds 2.[10,11] This is because the thermalization distance reaches 3-7 nm in organic condensed matters[12-14] and the reaction of the thermalized electrons with the molecules is a selective process.[15]

Owing to these efficient reaction mechanisms, chemically amplified resists excellently work not only for deep UV radiations but also for ionizing radiations. However, the thermalization distance becomes a concern with the reduction in feature size. The first $\beta$ tool of EUV lithography has already been shipped from ASML in 2010.[16] The extendibility of EUV lithography at half-pitches below $16 \mathrm{~nm}$ has recently attracted much attention. The shorter the thermalization distance is, the higher the resolution is expected to be. However, the chemically amplified resists discussed here are intended for use in high-volume production lines. Not only resolution but also LER and sensitivity are similarly important and cannot be neglected. The thermalization distance plays a critical role in the sensitization process, as described previously. In our previous study, the relationship between the thermalization distance and LER of 16-32 nm half-pitch was determined using two simple scaling models for latent image degradation and exposure dose dependence.[17] In this study, the optimum thermalization distance was investigated from the viewpoint of the RLS trade-off relationship by reaction-diffusion simulation. The effect of thermalization distance on acid image quality was clarified. On the basis of simulation results, the dependence of the optimum thermalization distance on half-pitch was discussed by extending the half-pitch from $16 \mathrm{~nm}$ discussed in the previous study to $11 \mathrm{~nm}$.

\section{Simulation method}

The formation of line-and-space patterns was simulated by changing the thermalization distance. The aerial image of incident EUV photons, I $(x$ : perpendicular to line pattern, $y$ : parallel to line pattern, $z$ : depth direction), was approximated using a cosine function.

$$
I(x, y, z)=A\left\{\frac{1}{2}\left[1+C \cos \left(\frac{\pi x}{p_{1 / 2}}\right)\right]\right\} \exp (-\alpha|z|)
$$

Here, $A, C, p_{1 / 2}$, and $\alpha$ represent the exposure dose, the contrast of the aerial image, the half-pitch of a line-and-space pattern, and the absorption coefficient of the resist, respectively. The details of the sensitization mechanisms of EUV resists have been reported elsewhere.[7,8,18] The resolution blur induced by secondary electrons[19-21] was incorporated using the spherically symmetric point-spread function for EUV resists.[22,23]

$$
\begin{aligned}
& P S F(r)=\frac{\phi_{\text {polymer }} N_{+} \int_{0}^{\infty} R_{A G} C_{A G} w d t}{\int_{r_{0}}^{\infty} w_{t=0} r^{2} d r} \\
& \frac{\partial w}{\partial t}=\frac{\partial^{2} w}{\partial r^{2}}+\left(\frac{2}{r}+\frac{S N_{+} e^{2}}{4 \pi \varepsilon_{0} \varepsilon k_{B} T r^{2}}\right) \frac{\partial w}{\partial r}-4 \pi R_{A G} C_{A G} w
\end{aligned}
$$

Here, $w, \phi_{\text {polymer }}, k_{\mathrm{B}}, T, R_{\mathrm{AG}}, C_{\mathrm{AG}}, e, \varepsilon_{0}, \varepsilon$, and $N_{+}$ represent the electron probability density, the deprotonation efficiency of radical cations, the Boltzmann constant, the absolute temperature, the effective reaction radius and concentration of the acid generators, the elementary electric charge, the dielectric constant in vacuum, the relative dielectric constant of the resist film, and the average number of positive charges generated by a single EUV photon, respectively. The initial condition for the diffusion equation [eq. (3)] is expressed as

$$
4 \pi w_{t=0} r^{2} d r=\frac{1}{r_{0}} \exp \left(-\frac{r}{r_{0}}\right) d r,
$$

where $r_{0}$ is the thermalization distance (the mean initial separation distance between a thermalized electron and its parent radical cation).[12-14] The acid distribution was calculated by convoluting the point-spread function with the aerial image of EUV photons. The preneutralization of acids before postexposure baking (PEB)[24-26] was assumed. Using the acid distribution after the preneutralization as a boundary condition, the subsequent reaction diffusion was calculated using the following equations.

$$
\begin{aligned}
& \frac{\partial C_{\text {acid }}}{\partial t}=\nabla\left(D_{\text {acid }} \nabla C_{\text {acid }}\right)-k_{N} C_{\text {acid }} C_{q} \\
& \frac{\partial C_{q}}{\partial t}=\nabla\left(D_{q} \nabla C_{q}\right)-k_{N} C_{\text {acid }} C_{q}
\end{aligned}
$$

Here, $C_{\text {acid }}, C_{\mathrm{q}}, D_{\text {acid }}, D_{\mathrm{q}}$, and $k_{\mathrm{N}}$ represent the concentrations of acids and quenchers, the diffusion constants of acids and quenchers, and the rate constant for neutralization, respectively. The deprotection was calculated as

$$
\frac{\partial C_{P}}{\partial t}=-k_{P} C_{\text {acid }} C_{P} \cdot
$$

Here, $C_{\mathrm{P}}$ and $k_{\mathrm{P}}$ represent the concentration of protected units and the rate constant for 
deprotection, respectively. As for the calculation of LER, it has been widely accepted that the main cause of LER in high-performance resists is the chemical inhomogeneity generated at the image boundary between the solubilized and insoluble regions of resist films (intermediate region).[27-29] Therefore, LER is proportional to the width of the intermediate region and can be approximated using the chemical gradient (the gradient of the protected unit concentration) as[30-32]

$$
L E R \approx \frac{f_{L E R}}{d m / d x} .
$$

Here, $f_{\text {LER }}, m$, and $\mathrm{d} m / \mathrm{d} x$ are a constant, the concentration of protected units normalized by its initial value, and the chemical gradient, respectively. Using the $f_{\text {LER }}$ of a typical chemically amplified EUV resist, SSR3,[5] LER was calculated.

The details of the point-spread function and the parameters used for calculation have been reported elsewhere.[17,22] TPS-tf was assumed as an acid generator. The parameters associated with a typical chemically amplified resist with a poly(4-hydroxystyrene) (PHS) backbone polymer and TPS-tf have been proved to reproduce the acid yields generated in resist films.[33,34] The absorption coefficient was set to be a typical value for chemically amplified EUV resists (3.8 $\mu \mathrm{m}^{-1}$ ).[33] The acid generator concentration was assumed to be constant during exposure. The error in the estimation of acid concentration caused by this assumption is less than 3\%.[35] This error does not significantly affect the following discussion. The rate constant for neutralization between acids and quenchers is equal to the diffusion-controlled rate.[36] The rate constant for deprotection, $k_{\mathrm{P}}$, was calculated as $k_{\mathrm{P}}=4 \pi R_{\mathrm{eff}} D$, where $R_{\text {eff }}$ and $D$ are the effective reaction radius and the sum of the diffusion constants of related molecules. The effective reaction radius has been investigated for a typical chemically amplified EUV resist and estimated to be approximately 0.1 nm.[31,32] The initial protection ratio of the polymer was $30 \%$. In chemically amplified resists used for ionizing radiation, the protons of acids are generated through the deprotonation of polymer radical cations. [37,38] The hydroxyl groups are the major proton source.[39-42] The protection of hydroxyl groups has been reported to reduce acid yields.[37,43] Although the deprotonation efficiency depends on the molecular structures of the protecting groups, the deprotonation efficiency of the protected units was set to be a typical value of 0.3.[43] The diffusion constants of acids and quenchers were assumed to be $1 \mathrm{~nm}^{2} \mathrm{~s}^{-1}$. The quencher diffusion constant was assumed to be the same as the acid diffusion constant. The effect of the quencher diffusion constant has been reported and this assumption does not significantly affect the following discussion.[44] The effect of matrix-dependent diffusion on the chemical gradient has also been reported.[45,46] The half-pitch was changed from 11 to $32 \mathrm{~nm}$. The resist thicknesses were assumed to be $25 \mathrm{~nm}$ for 11 $\mathrm{nm}$ half-pitch, $35 \mathrm{~nm}$ for $16 \mathrm{~nm}$ half-pitch, $50 \mathrm{~nm}$ for $23 \mathrm{~nm}$ half-pitch, and $70 \mathrm{~nm}$ for $32 \mathrm{~nm}$ half-pitch. The contrast of the aerial image was assumed to be 1 for simplicity. The effect of the contrast on the chemical gradient has also been reported.[3,47] The contribution of the direct electronic excitation of acid generators to acid generation was incorporated using the $f_{\text {excitation }}$ factor $\left(1.4 \times 10^{-2} \mathrm{dm}^{3} \mathrm{~mol}^{-1} \mathrm{eV}^{-1}\right)$. [48] The other parameters associated with basic material properties were determined mainly by the quantitative analysis of acid yields generated in model resists upon exposure to EUV and an electron beam.[49]

\section{Results and discussion}

Figure 1 shows the profiles of acid images in the direction vertical to the line pattern. The half-pitch of the line-and-space pattern is $16 \mathrm{~nm}$. Although the EUV intensity at $x=16 \mathrm{~nm}$ (the center of the line pattern) is 0 as expressed by eq. (1), the acid concentration is not 0 at the same position. This is the effect of the resolution blur induced by secondary electrons. At the

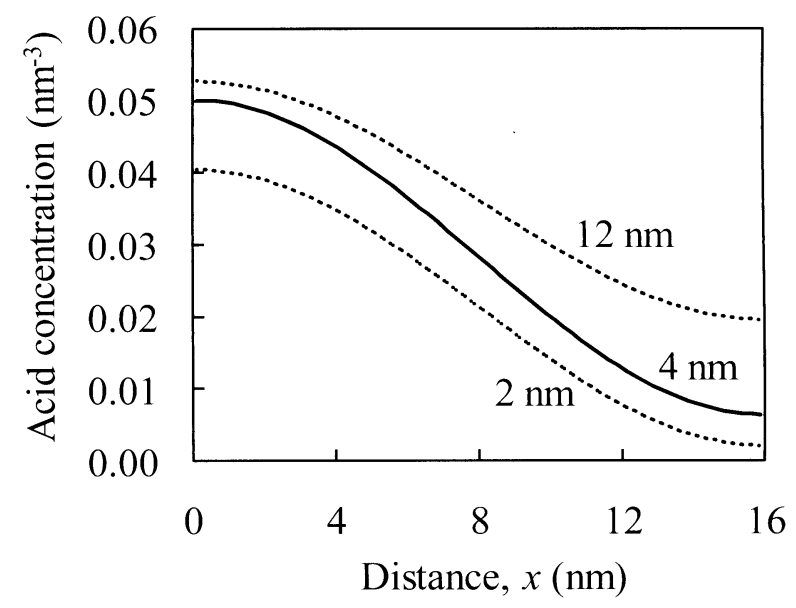

Fig. 1. Dependence of acid distribution on thermalization distance. The values indicated in the graph in $\mathrm{nm}$ represent the thermalization distance. The exposure dose was $10 \mathrm{~mJ} \mathrm{~cm}$. The half-pitch was $16 \mathrm{~nm}$. The acid generator concentration was 10 $w t \%$. 
thermalization distance $r_{0}$ of $2 \mathrm{~nm}$, the increase in acid concentration at $x=16 \mathrm{~nm}$ is negligible. The acid concentration at $x=16 \mathrm{~nm}$ increases with thermalization distance. The contrast of the EUV aerial image is 1 . The contrasts of the acid images are 0.90 at $r_{0}=2 \mathrm{~nm}, 0.77$ at $r_{0}=4 \mathrm{~nm}$, and 0.46 at $r_{0}=12 \mathrm{~nm}$. The resolution blur derived from the thermalization distance results in the degradation of the acid images as expected. However, the quantum efficiency of acid generation also increases with thermalization distance. The acid concentration at $x=0 \mathrm{~nm}$, namely, the center of the space, increases with thermalization distance. Because of these two different effects, the slope at $r_{0}=4 \mathrm{~nm}$, namely, the intended boundary between the space and the line, is steeper at $x=8 \mathrm{~nm}$ than those at $r_{0}=2$ and $12 \mathrm{~nm}$.

The subsequent reaction diffusion during PEB was calculated using the acid concentration profiles shown in Fig. 1. The PEB time, dissolution point, and quencher concentration were optimized to maximize the chemical gradient (the gradient of protected unit concentration) at $x=8 \mathrm{~nm}$. This condition is equivalent to the condition indicating that LER is minimized because LER is inversely proportional to the chemical gradient as expressed by eq. (8). The calculated protected unit concentration profiles are shown in Fig. 2. The chemical gradient at $r_{0}=4 \mathrm{~nm}$ is higher at $x=8 \mathrm{~nm}$ than those at $r_{0}=2$ and $12 \mathrm{~nm}$. Therefore, the LER at $r_{0}=4 \mathrm{~nm}$ is expected to be lower than those at $r_{0}$ $=2$ and $12 \mathrm{~nm}$.

Similarly, the latent images of line-and-space patterns were calculated by changing the half-pitch, thermalization distance, and acid generator concentration. The relationship between LER and thermalization distance is shown in Fig. 3. LER increases with a reduction in half-pitch as reported by many researchers. LER decreases with an increase in acid generator concentration because the quantum efficiency of acid generation increases with acid generator concentration. As for thermalization distance, LER decreases and then increases with an increase in thermalization distance. The optimum thermalization distances determined on the basis of the RLS trade-off relationship are $6-7 \mathrm{~nm}$ at $32 \mathrm{~nm}$ half-pitch, $5 \mathrm{~nm}$ at $23 \mathrm{~nm}$ half-pitch, $4 \mathrm{~nm}$ at $16 \mathrm{~nm}$ half-pitch, and $3 \mathrm{~nm}$ at $11 \mathrm{~nm}$ half-pitch. At $32 \mathrm{~nm}$ half-pitch, LER does not depend much on thermalization distance. Rather, the increase in LER at a short thermalization distance is prominent. This indicates that the effect of thermalization distance on

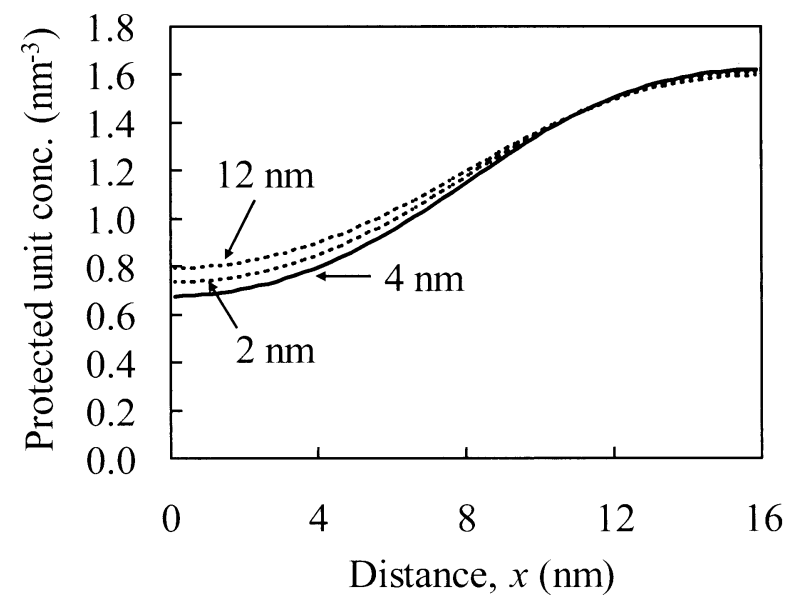

Fig. 2. Dependence of protected unit distribution on thermalization distance. The numerical values indicated in the graph in $\mathrm{nm}$ represent the thermalization distance. The process parameters, such as PEB time and quencher concentration, were optimized for each thermalization distance to obtain the highest chemical gradient at the intended image boundary. The exposure dose was $10 \mathrm{~mJ} \mathrm{~cm}^{-2}$. The half-pitch was $16 \mathrm{~nm}$. The acid generator concentration was $10 \mathrm{wt} \%$.

quantum efficiency is more important than that on the resolution blur at $32 \mathrm{~nm}$ half-pitch from the viewpoint of resist performance. With the reduction in half-pitch, the effect of thermalization distance becomes evident and a short thermalization distance is required to reduce the LER. However, the thermalization distance of $3 \mathrm{~nm}$ is sufficient even at $11 \mathrm{~nm}$ half-pitch. The thermalization distances in PHS and acrylate polymers have been reported to be 3-4 and 5-7 nm, respectively.[23,38,50] From the viewpoint of thermalization distance, acrylate polymers have an advantage over PHS at 23-32 nm half-pitch. PHS has an advantage over acrylate polymers at 11-16 $\mathrm{nm}$ half-pitch. Note that the dependence of LER on thermalization distance can be suppressed by increasing the acid generator concentration, as seen in Fig. 3.

\section{Conclusion}

The thermalization distance of secondary electrons plays a critical role in the sensitization of chemically amplified resists used for ionizing radiations, such as EUV radiation. The thermalization distance has both positive and negative effects on the resist performance. Therefore, an optimum thermalization distance exists and depends on feature size. The optimum thermalization distance of line-and-space patterns with $11 \mathrm{~nm}$ half-pitch was $3 \mathrm{~nm}$. Because the 


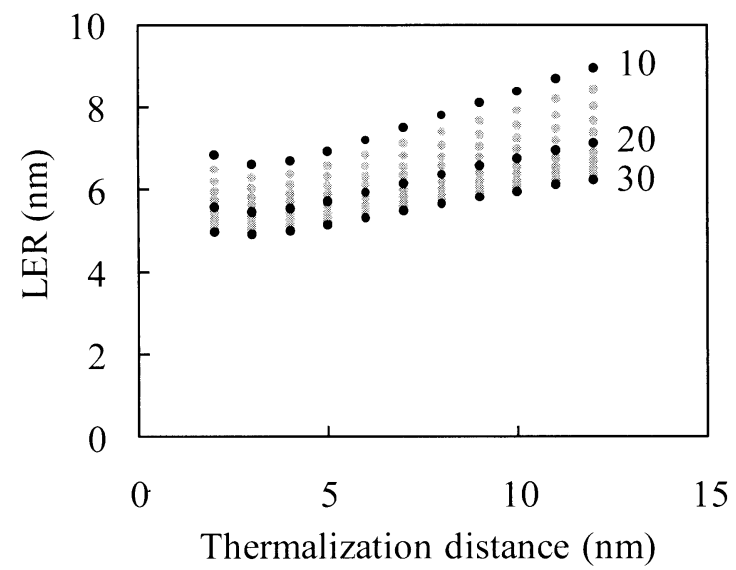

(a) $11 \mathrm{~nm}$

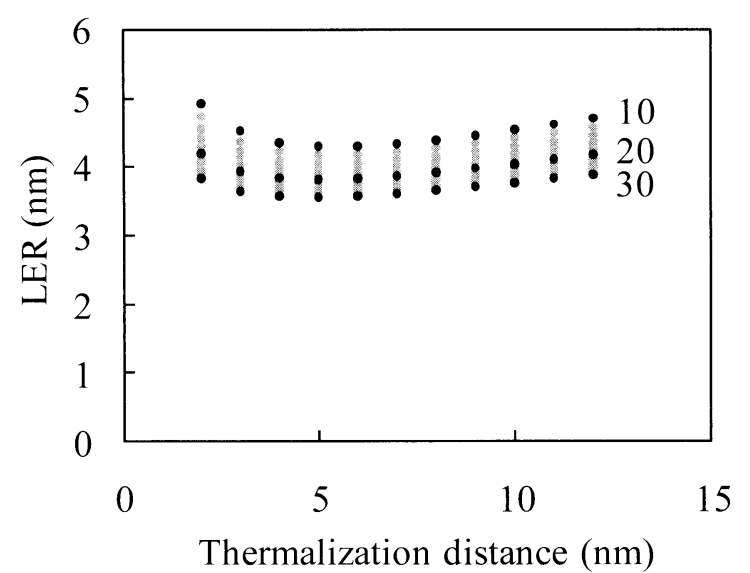

(c) $23 \mathrm{~nm}$

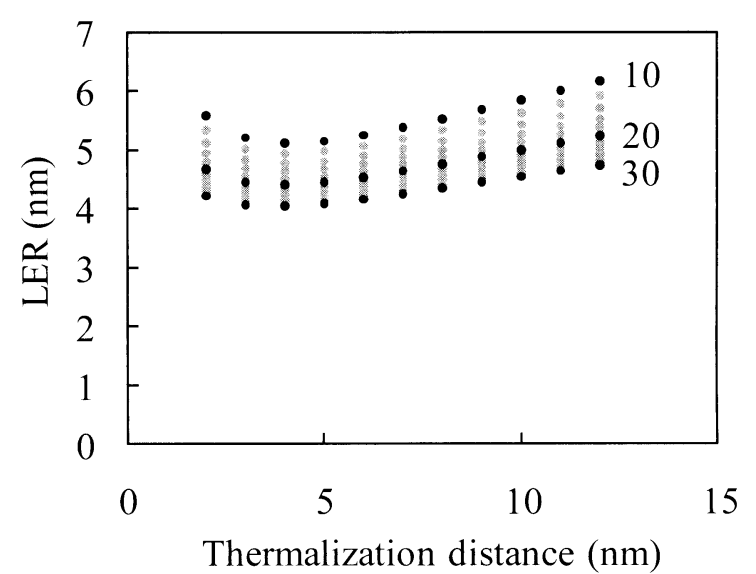

(b) $16 \mathrm{~nm}$

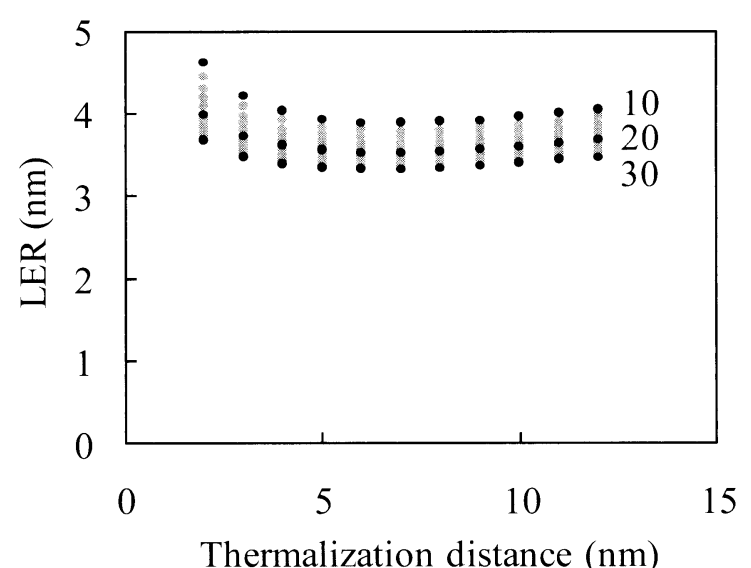

(d) $32 \mathrm{~nm}$

Fig. 3. Relationship between thermalization distance and LER. The half-pitch was changed from (a) $11 \mathrm{~nm}$ to (d) $32 \mathrm{~nm}$. The acid generator concentration was changed from 10 to $30 \mathrm{wt} \%$ in a step of $2 \mathrm{wt} \%$. The numerical values indicated in the graphs denote the acid generation concentration in $\mathrm{wt} \%$. The exposure dose was $10 \mathrm{~mJ} \mathrm{~cm}^{-2}$. $f_{\mathrm{LER}}$ was assumed to be 0.3 .

thermalization distance of current typical polymer films is $3-4 \mathrm{~nm}$, further reduction in thermalization distance is not necessary at half-pitches above 11 $\mathrm{nm}$ as long as the strict sensitivity requirement is imposed on the resist materials.

\section{Acknowledgement}

This work was supported in part by a Grant-in-Aid for Scientific Research (Project No. 21656238) from the Ministry of Education, Culture, Sports, Science and Technology of Japan (MEXT).

\section{References}

1. H. Ito, Microlithography/Molecular Imprinting (Springer, Heidelberg, 2005) Advances in Polymer Science Series, Vol. 172, p. 37.

2. G. M. Gallatin, Proc. SPIE, 5754 (2005) 38.

3. T. Kozawa and S. Tagawa, J. Photopolym. Sci. Technol., 22 (2009) 51.

4. S. Wurm, Jpn. J. Appl. Phys., 46 (2007) 6105.

5. T. Itani, Microelectron. Eng., 86 (2009) 207.
6. P. P. Naulleau, C. N. Anderson, J. Chiu, K. Dean, P. Denham, S. George, K. A. Goldberg, B. Hoef, G. Jones, C. Koh, B. La Fontaine, A. Ma, W. Montgomery, D. Niakoula, J. Park, T. Wallow, and S. Wurm, J. Vac. Sci. Technol. B, 27 (2009) 66.

7. T. Kozawa, S. Tagawa, H. Oizumi, and I. Nishiyama, J. Vac. Sci. Technol. B, 24 (2006) L27.

8. T. Kozawa, Y. Yoshida, M. Uesaka, and S. Tagawa, Jpn. J. Appl. Phys., 31 (1992) 4301.

9. S. Tanuma, C. J. Powell, and D. R. Penn, Surf. Interface Anal., 21 (1993) 165.

10. R. L. Brainard, P. Trefonas, J. H. Lammers, C. A. Cutler, J. F. Mackevich, A. Trefonas, and S. A. Robertson, Proc. SPIE, 5374 (2004) 74.

11. R. Hirose, T. Kozawa, S. Tagawa, T. Kai, and T. Shimokawa, Appl. Phys. Express, 1 (2008) 027004.

12. A. Saeki, T. Kozawa, Y. Yoshida, and S. Tagawa, Jpn. J. Appl. Phys., 41 (2002) 4213. 
13. K. Okamoto, A. Saeki, T. Kozawa, Y. Yoshida, and S. Tagawa, Chem. Lett., 32 (2003) 834.

14. K. Okamoto, T. Kozawa, A. Saeki, Y. Yoshida, and S. Tagawa, Radiat. Phys. Chem., 76 (2007) 818.

15. T. Kozawa, A. Saeki, Y. Yoshida, and S. Tagawa, Jpn. J. Appl. Phys., 41 (2002) 4208.

16. R. de Leeuw, C. Wagner, R. Peeters, S. Young, N. Harned, M. Lowisch, H. Meiling, D. Ockwell, E. van Setten, and J. Stoeldraijer, presented at EUV Symp., 2010, ET-01.

17. T. Kozawa and S. Tagawa, Jpn. J. Appl. Phys., 50 (2011) 036505.

18. T. Kozawa, S. Tagawa, H. B. Cao, H. Deng, and M. J. Leeson, J. Vac. Sci. Technol. B, 25 (2007) 2481.

19. T. Kozawa, H. Yamamoto, A. Saeki, and S. Tagawa, J. Vac. Sci. Technol. B, 23 (2005) 2716.

20. T. Kozawa and S. Tagawa, J. Appl. Phys., 99 (2006) 054509.

21. T. Kozawa, S. Tagawa, and M. Shell, J. Appl. Phys., 103 (2008) 084306.

22. T. Kozawa, A. Saeki, and S. Tagawa, Appl. Phys. Express, 1 (2008) 027001.

23. T. Kozawa, A. Saeki, and S. Tagawa, J. Vac. Sci. Technol. B, 22 (2004) 3489.

24. K. Natsuda, T. Kozawa, K. Okamoto, and S. Tagawa, Jpn. J. Appl. Phys., 45 (2006) L1256.

25. K. Natsuda, T. Kozawa, K. Okamoto, and S. Tagawa, Jpn. J. Appl. Phys., 46 (2007) 7285.

26. T. Kozawa, S. Tagawa, J. J. Santillan, M. Toriumi, and T. Itani, Jpn. J. Appl. Phys., 47 (2008) 4926.

27. W. Hinsberg, F. A. Houle, J. Hoffnagle, M. I. Sanchez, G. M. Wallraff, M. Morrison, and S. Frank, J. Vac. Sci. Technol. B, 16 (1998) 3689.

28. S. C. Palmateer, S. G. Cann, J. E. Curtin, S. P. Doran, L. M. Eriksen, A. R. Forte, R. R. Kunz, T. M. Lyszczarz, M. B. Stern, and C. M. Nelson-Thomas, Proc. SPIE, 3333 (1998) 634.

29. T. B. Michaelson, A. R. Pawloski, A. Acheta, Y. Nishimura, and C. G. Willson, Proc. SPIE, 5753 (2005) 368 .

30. T. Kozawa, H. Oizumi, T. Itani, and S. Tagawa, Appl. Phys. Express, 3 (2010) 036501.

31. T. Kozawa, H. Oizumi, T. Itani, and S. Tagawa, Jpn. J. Appl. Phys., 49 (2010) 066504.

32. T. Kozawa, H. Oizumi, T. Itani, and S. Tagawa, Jpn. J. Appl. Phys., 49 (2010) 116505.
33. R. Hirose, T. Kozawa, S. Tagawa, T. Kai, and T. Shimokawa, Jpn. J. Appl. Phys., 46 (2007) L979.

34. R. Hirose, T. Kozawa, S. Tagawa, D. Shimizu, T. Kai, and T. Shimokawa, Jpn. J. Appl. Phys., 47 (2008) 7125.

35. T. Kozawa, S. Tagawa, and M. Shell, Jpn. J. Appl. Phys., 46 (2007) L1143.

36. W. D. Hinsberg, F. A. Houle, M. I. Sanchez, and G. M. Wallraff, IBM J. Res. Dev., 45 (2001) 667.

37. T. Kozawa, S. Nagahara, Y. Yoshida, S. Tagawa, T. Watanabe, and Y. Yamashita, J. Vac. Sci. Technol. B, 15 (1997) 2582.

38. T. Kozawa, S. Tagawa, T. Kai, and T. Shimokawa, J. Photopolym. Sci. Technol., 20 (2007) 577.

39. H. Yamamoto, T. Kozawa, A. Nakano, K. Okamoto, Y. Yamamoto, T. Ando, M. Sato, H. Komano, and S. Tagawa, Jpn. J. Appl. Phys., 43 (2004) L848.

40. H. Yamamoto, A. Nakano, K. Okamoto, T. Kozawa, and S. Tagawa, Jpn. J. Appl. Phys., 43 (2004) 3971.

41. A. Nakano, T. Kozawa, K. Okamoto, S. Tagawa, T. Kai, and T. Shimokawa, Jpn. J. Appl. Phys., 45 (2006) 6866.

42. H. Yamamoto, T. Kozawa, S. Tagawa, H. B. Cao, H. Deng, and M. J. Leeson, Jpn. J. Appl. Phys., 46 (2007) L142.

43. H. Yamamoto, T. Kozawa, A. Nakano, K. Okamoto, S. Tagawa, T. Ando, M. Sato, and H. Komano, Jpn. J. Appl. Phys., 44 (2005) 5836.

44. T. Kozawa, S. Tagawa, J. J. Santillan, and T. Itani, Jpn. J. Appl. Phys., 47 (2008) 5404.

45. T. Kozawa, S. Tagawa, J. J. Santillan, and T. Itani, J. Photopolym. Sci. Technol., 21 (2008) 421.

46. T. Kozawa, H. Oizumi, T. Itani, and S. Tagawa, Jpn. J. Appl. Phys., 49 (2010) 036506.

47. T. Kozawa and S. Tagawa, Appl. Phys. Express, 2 (2009) 095004.

48. T. Kozawa, T. Shigaki, K. Okamoto, A. Saeki, S. Tagawa, T. Kai, and T. Shimokawa, J. Vac. Sci. Technol. B, 24 (2006) 3055.

49. T. Kozawa and S. Tagawa, Jpn. J. Appl. Phys., 49 (2010) 030001.

50. T. Kozawa and S. Tagawa, Jpn. J. Appl. Phys., 50 (2011) 030209. 